\title{
Biological Properties of Ti-Nb-Zr-O Nanostructures Grown on Ti35Nb5Zr Alloy
}

\author{
Zhaohui Li, ${ }^{1}$ Congqin Ning, ${ }^{2}$ Dongyan Ding, ${ }^{1}$ Hegang Liu, ${ }^{1}$ and Lin Huang, ${ }^{1,2}$ \\ ${ }^{1}$ Institute of Microelectronic Materials and Technology, School of Materials Science and Engineering, \\ Shanghai Jiao Tong University, Shanghai 200240, China \\ ${ }^{2}$ State Key Laboratory of High Performance Ceramics and Superfine Microstructure, Shanghai Institute of Ceramics, \\ Chinese Academy of Sciences, Shanghai 200050, China \\ Correspondence should be addressed to Dongyan Ding, dyding@sjtu.edu.cn
}

Received 25 May 2012; Accepted 20 August 2012

Academic Editor: Cui ChunXiang

Copyright ( $) 2012$ Zhaohui Li et al. This is an open access article distributed under the Creative Commons Attribution License, which permits unrestricted use, distribution, and reproduction in any medium, provided the original work is properly cited.

Surface modification of low modulus implant alloys with oxide nanostructures is one of the important ways to achieve favorable biological behaviors. In the present work, amorphous Ti-Nb-Zr-O nanostructures were grown on a peak-aged Ti35Nb5Zr alloy through anodization. Biological properties of the Ti-Nb-Zr-O nanostructures were investigated through in vitro bioactivity testings, stem cell interactions, and drug release experiments. The Ti-Nb-Zr-O nanostructures demonstrated a good capability of inducing apatite formation after immersion in simulated body fluids (SBFs). Drug delivery experiment based on gentamicin and the Ti-Nb-Zr-O nanostructures indicated that a high drug loading content could result in a prolonged release process and a higher quantity of drug residues in the oxide nanostructures after drug release. Quick stem cell adhesion and spreading, as well as fast formation of extracellular matrix materials on the surfaces of the Ti-Nb-Zr-O nanostructures, were found. These findings make it possible to further explore the biomedical applications of the Ti-Nb-Zr-O nanostructure modified alloys especially clinical operation of orthopaedics by utilizing the nanostructures-based drug-release system.

\section{Introduction}

In recent decades, skeletal diseases have increased with extending of human life expectancy. Severe open fracture and soft tissue injury of limb skin are common in cases of clinical orthopedics. During the clinical orthopedic treatment, orthopedic infections often take place in trauma, surgery or after in vivo implantation. And some infections may not be cured for a long time. For the osteomyelitis caused by virus infection a slow drug-release at fixed-point is an important biomedical issue. An ideal local antibiotic delivery is referred to stable and lasting drug concentrations, avoiding a lower local concentration and a higher serum concentration caused by systemic administration and a series of adverse reactions. Therefore, it is of great interest for researchers and clinicians to develop high performance implanting materials for artificial bone tissue repair, which can fill the bone defect and simultaneously have a slow release of drugs at local areas to fight infection.
For biomedical applications $\beta$-type titanium alloys such as $\mathrm{Ti}-\mathrm{Nb}$ and $\mathrm{Ti}-\mathrm{Nb}-\mathrm{Zr}$ alloy systems have aroused great interests [1-3] mainly due to their nontoxic alloying design [4], lower elastic modulus compared to traditional alloys such as Ti-6Al-4V [5], good biocompatibility and better corrosion resistance [6]. However, bonding with bone in a simple interlocking way is the primary reason obstructing applications of $\mathrm{Ti}$ and $\mathrm{Ti}$ alloys in orthopedic and dental implants because a nonchemical bonding can result in the loosening of an implant and eventual failure of the implantation [7]. Therefore, fabrication of a stable oxide nanostructures on $\mathrm{Ti}$ metal and alloys has been expected to improve the bonding behavior [8]. Briggs et al. reported the formation of nanoporous oxide layer on the surface of titanium alloys and they observed flattened cells with filopodia attaching to the pores with a good coverage [9].

Several research groups have reported the anodic formation of oxide nanostructures on the surfaces of titanium alloys to achieve a good bioactivity and cell culture as well 
as nanostructured drug-release system [10-15]. And the superiority of oxide nanotubes grown on Ti metals or alloys over flat Ti surfaces has been proved with regard to osteointegration of an implant material and adhesion/proliferation of cultured cells $[8,16]$.

Anodization of $\mathrm{Ti}$ alloys to fabricate doped $\mathrm{TiO}_{2}$ nanotubes has been widely reported in literature. Ghicov et al. reported the anodization of Ti45Nb alloy to grow Ti$\mathrm{Nb}-\mathrm{O}$ nanotubes [17]. Qin et al. reported the anodization of single-phase Ti35 $\mathrm{Nb}$ alloy to form $\mathrm{Nb}$-doped titania nanotubes with a good sensing performance [18]. Bai et al. successfully realize a uniform anodization of biphase Ti$35 \mathrm{Nb}-5 \mathrm{Zr}$ alloy in nonaqueous glycerol-based electrolytes to grown nanotube arrays [19].

To date, biological properties of Ti-Nb-Zr-O nanostructures grown on low modulus Ti-35Nb-5Zr alloy have not been reported. Thus, in the present work, peakaged Ti35Nb5Zr alloy with a biphase microstructure was anodized in an aqueous electrolyte to grown Ti-Nb-Zr$\mathrm{O}$ nanostructures on the alloy surface. In vitro bioactivity, drug-release properties and mesenchymal stem cell (MSC) adhesion behaviors of the Ti-Nb-Zr-O nanostructures were investigated.

\section{Experimental Details}

2.1. Fabrication of Ti-Nb-Zr-O Nanotubes. Peak-aged Ti35Nb5Zr alloy plates with a size of $10 \mathrm{~mm} \times 10 \mathrm{~mm} \times$ $1 \mathrm{~mm}$ were grinded and polished with 2000\# SiC emery papers, and then ultrasonically cleaned with absolute alcohol. Finally they were rinsed with deionized water and dried in a $\mathrm{N}_{2}$ stream. Electrochemical anodization was carried out with a DC voltage stabilizer. All of the samples were fabricated at $15 \mathrm{~V}$ (for one and a half hours) in $1 \mathrm{M}\left(\mathrm{NH}_{4}\right)_{2} \mathrm{SO}_{4}$ solution containing $0.5 \mathrm{wt} \% \mathrm{NH}_{4} \mathrm{~F}$. After anodization, the samples were rinsed with deionized water and dried in an $\mathrm{N}_{2}$ stream.

2.2. In Vitro Bioactivity. We evaluated the in vitro bioactivity of the as-anodized samples in terms of their capability in inducing apatite formation in simulated body fluid (SBF). The anodized samples were soaked in the $\mathrm{SBF}$ at $37^{\circ} \mathrm{C}$ for different soaking times. During the in vitro testing process, the SBF was refreshed every day. After a predetermined soaking time of 60 hours or 7 days, the samples were taken out of the SBF, rinsed with deionized water, and finally dried in air for further surface examination.

2.3. In Vitro Drug Delivery. We used a broad-spectrum antibiotic of gentamicin for the investigation of drug-release behavior of the Ti-Nb-Zr-O nanostructures. Before drug loading, the nanostructure samples were rinsed with deionized water and dried in an $\mathrm{N}_{2}$ stream. The gentamicin was loaded to the oxide nanostructures through a lyophilization method. Firstly, the nanostructured surface was pipetted on by one microliter of gentamicin solution $(40 \mathrm{mg} / \mathrm{mL})$ to allow the antibiotic gently to spread onto and cover the whole surface. The samples were then dried under vacuum at room temperature for $2 \mathrm{~h}$. After the surface of the samples was



Figure 1: TEM image of the peak-aged Ti35Nb5Zr alloy showing bi-phase microstructure of needle-like $\alpha$-phase formed in the $\beta$ matrix.

dried, the drug loading step was repeated until the drugloaded content was sufficient for the nanostructured surface of the anodized samples. After accomplishment of the final step of drying, the drug-loaded samples were rinsed with phosphate buffered saline (PBS) solution to remove redundant drugs on the nanostructured surface of the samples. The content of drug loading was obtained through calculating the concentration difference of gentamicin between the initial drug solution and PBS solution after rinsing the drug-loaded samples.

The drug-loaded samples were placed in the Eppendorf centrifuge tubes containing one milliliter PBS solution and the samples were soaked in the PBS solution. The centrifuge tubes were oscillated by the thermostatic water bath oscillator at $37^{\circ} \mathrm{C}$. Each time 200 microlitre solution was removed from the centrifuge tubes and another 200 microlitre fresh PBS solution was added to the centrifuge tubes at predetermined intervals. The concentration dilution of PBS solution in Eppendorf centrifuge tubes resulting from the addition of fresh PBS solution cannot be neglected when the release kinetics of loading drugs were investigated. Finally, all of the extraction solutions were collected and analyzed with UV/VIS spectroscopy for drug content.

2.4. Cell Adhesion. Mouse bone marrow MSCs (mMSC) were cultured and passaged. Cells from the fourth passage were used for the cell interaction with Ti-Nb-Zr-O nanostructures. The as-anodized samples were autoclaved and then put in a 24-well plate. One milliliter of cell suspension with a cell density of $2 \times 105$ cells $\mathrm{mL}^{-1}$ was added to each well. After different culture times (2, 12 and $24 \mathrm{~h})$, the cultured samples were treated by a series of steps reported in our previous work [14]. For comparison, MSCs were also cultured on the surface of pure Ti plates.

Microstructure of the alloy substrate was investigated with transmission electron microscope (TEM). Surfaces of the above as-anodized, SBF-treated and cell-attached samples were characterized with scanning electron microscope 


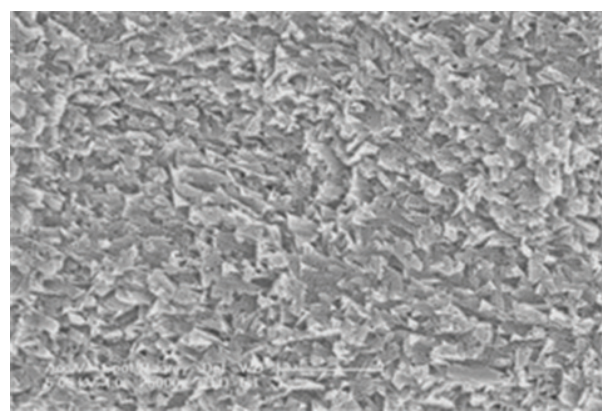

(a)

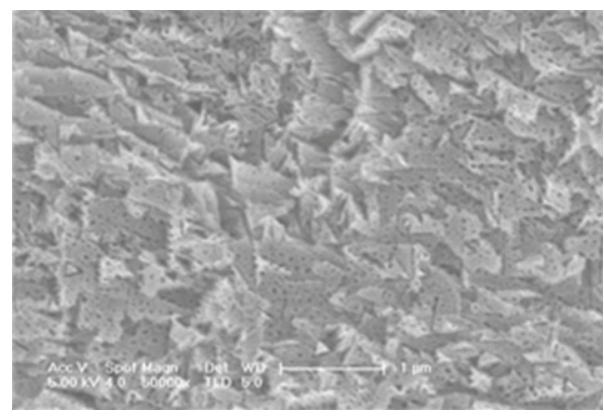

(b)

FIgURE 2: Surface morphology of the as-anodized Ti35Nb5Zr alloy (a) low magnification and (b) high magnification images.

(SEM; FEI SIRION 200) and energy dispersive X-ray analysis (EDXA; OXFORD INCA).

\section{Results and Discussion}

3.1. Anodic Oxide Grown on Ti35Nb5Zr Alloy. Figure 1 presents TEM images of the peak-aged Ti35Nb5Zr alloy. It could be found that the peak-aged alloy consisted of biphase microstructures with needle-like $\alpha$-phase precipitation formed in the $\beta$ matrix. Figure 2 shows surface morphologies of the as-anodized Ti35Nb5Zr alloy sample. As shown in Figure 2, large quantity of needle-like grooves together with quite a few nanotubular structures could be found. It was obvious that the as-grown anodic oxide was a kind of mixed nanostructures resulting from a phase-dependent anodization (uneven anodization at different phase regions) in the as-mentioned aqueous electrolytes. The needle-like grooves on the nanostructured surface mainly resulted from the much quicker dissolution rate of the $\alpha$-phase precipitations compared to the $\beta$-phase matrix during the anodization process [20]. The nanotubular structures with a pore size of around $60 \mathrm{~nm}$ should correspond to the $\beta$-phase matrix. The thickness of the anodic layer was so thin that a cross-sectional image was not available due to uneasy mechanical fracture/separation of the anodic oxide layer.

EDX analyses of the as-grown Ti-Nb-Zr-O oxide reveals that the weight percentage of the $\mathrm{Nb}, \mathrm{Zr}$, and $\mathrm{O}$ elements in the nanotubes is about $29.3 \%, 2.4 \%$, and $37.4 \%$, respectively. $\mathrm{X}$-ray diffraction of the as-anodized samples indicates that the as-grown $\mathrm{Ti}-\mathrm{Nb}-\mathrm{Zr}-\mathrm{O}$ oxide nanostructures are amorphous. As a high-temperature heat treatment may do damage to mechanical properties of the nanotube/alloy interface, we directly use the above amorphous nanostructures for the following biological evaluations.

3.2. In Vitro Bioactivity of the Ti-Nb-Zr-O Nanostructures. We evaluate the in vitro bioactivity of the as-anodized samples through examining the apatite formation on the Ti-Nb$\mathrm{Zr}-\mathrm{O}$ nanostructures. Figure 3 presents surface morphologies of the $\mathrm{Ti}-\mathrm{Nb}-\mathrm{Zr}-\mathrm{O}$ nanostructure samples immersed in SBF for 60 hours or 7 days. After 60 hours of immersion, a large quantity of apatite deposition could be found on the surface of the Ti-Nb-Zr-O nanostructures (Figures 3(a) and 3(b)). Nearly half of the top surfaces of the Ti-Nb-ZrO oxides were covered with worm-like apatite depositions. With increase of the immersion time to 7 days, the quantity of apatite deposition remarkably increased (Figure 3(c)). The nanostructured surfaces were completely covered with apatite deposition. The deposition layer was much thicker than that of the 60-hour system. Similar to the apatite formation on Ti-Al-V-O and Ti-Nb-O nanostructures, our EDXA pattern indicated that the deposition on the nanotubes is a kind of carbonated apatite which contains three main elements of a typical apatite-like deposition including calcium, phosphorus, and oxygen elements as well as a large amount of carbon element $[14,21]$. These experimental results indicate that the Ti-Nb-Zr-O nanostructures could induce considerable apatite formation within 60 hours of immersion.Therefore, the Ti-Nb-Zr-O oxide nanostructures could improve the in vitro bioactivity of Ti35Nb5Zr alloy, which is favorable for further formation of bone tissues in vivo.

\subsection{Drug-Release Behavior of Ti-Nb-Zr-O Nanostructures.} The Ti-Nb-Zr-O nanostructures served as a container during loading the drugs. The two-dimension size of a gentamicin molecular was around $0.52 \times 1.53 \mathrm{~nm}^{2}$ [22]. Therefore, the gentamicin molecular could easily get into the Ti-Nb$\mathrm{Zr}-\mathrm{O}$ nanopores and nanogrooves. A larger nanopores or nanogrooves may result in a higher drug loading content.

The drug loading content was obtained through calculation of the concentration difference between the initial gentamicin solution and phosphate buffered saline (PBS) solution after rinsing the drug-loaded samples. And the efficiency of diverse drug loading content was simultaneously determined. Figure 4 shows the drug loading efficiency for the drug loading contents of $400 \mu \mathrm{g}, 600 \mu \mathrm{g}$, and $800 \mu \mathrm{g}$. This group of data indicates that the loading efficiency of gentamicin was between $15 \%$ and $46 \%$. The drug loading efficiency was enhanced with increase of the drug loading content.

During the drug release process we calculated and fitted the drug release curves according to the concentration of gentamicin in the solution which was taken out from Eppendorf centrifuge tubes at different times. The release kinetics corresponding to the three kinds of drug loading contents was studied as a function of time. The drug release curves shown in Figure 5 reveal that the release process with 


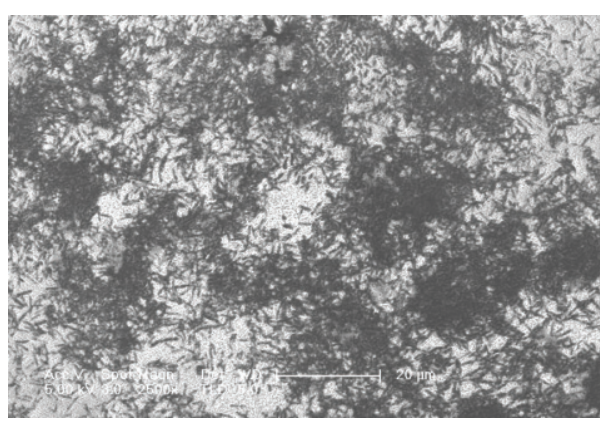

(a)

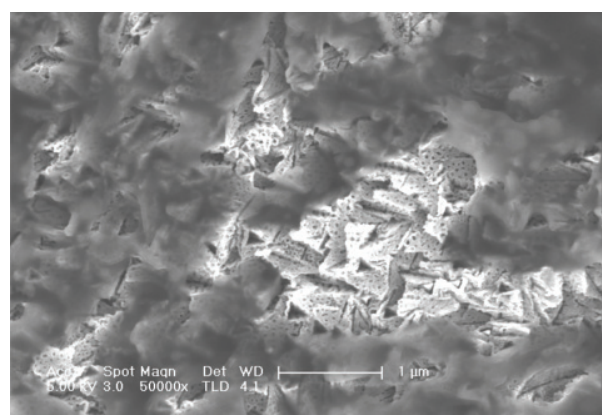

(b)

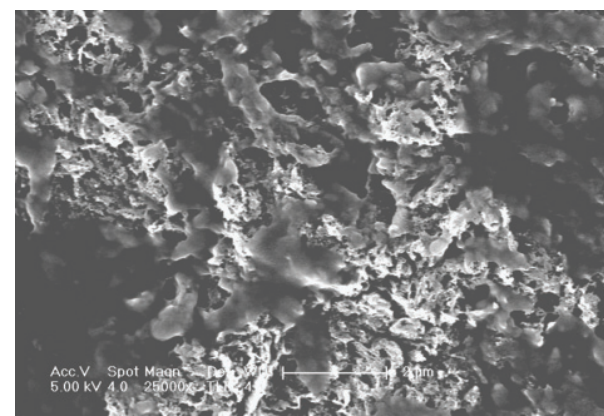

(c)

FIGURE 3: Surface morphologies of the Ti-Nb-Zr-O nanostructures after immersion in SBF for different time (a)-(b) 60 hours and (c) 7 days.

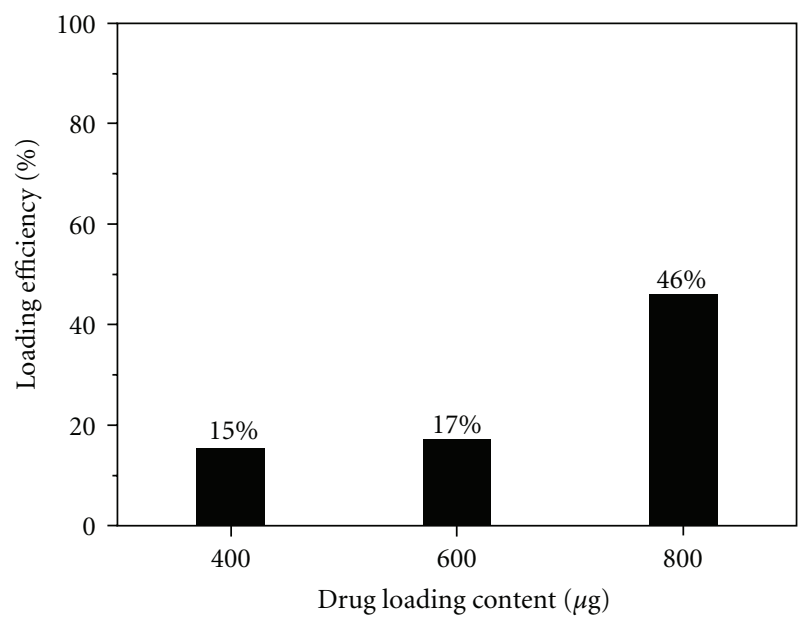

Figure 4: Drug loading efficiency of the Ti-Nb-Zr-O nanostructures.

the drug loading contents of $400 \mu \mathrm{g}$ and $600 \mu \mathrm{g}$ lasted for 36 minutes and 48 minutes, respectively. Whereas, for the largest drug loading content of $800 \mu \mathrm{g}$, the corresponding release process lasted for 105 minutes. This indicates that the release process of the high-content drug loading system is slower than that of the low-content drug loading system. Moreover, from the view of the drug release efficiency, the higher is the drug loading content, the more drug residues are left in the Ti-Nb-Zr-O nanostructures. The above drugrelease behavior of the $\mathrm{Ti}-\mathrm{Nb}-\mathrm{Zr}-\mathrm{O}$ nanostructures should be

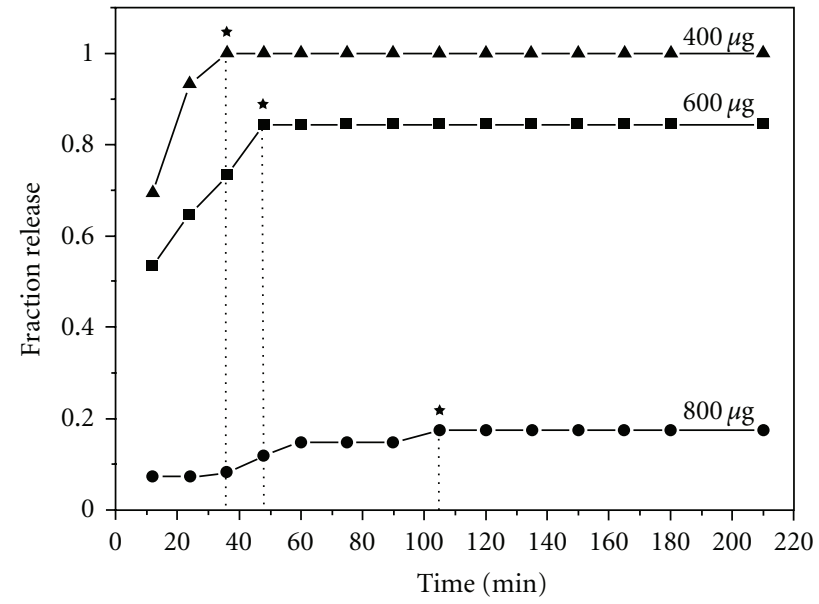

Figure 5: Drug release kinetics of the drug-loaded Ti-Nb-Zr-O nanostructures as a function of time.

further investigated for a local drug release during the clinical operation of orthopaedics.

3.4. Interaction of Stem Cells with Ti-Nb-Zr-O Nanostructures. Figure 6 presents SEM images of stem cells attached on the Ti-Nb-Zr-O nanostructures after different culture times. As can be seen in Figures 6(a) and 6(b), after a 2-hour cell culture, a large quantity of high-density stem cells attached and spread on the nanostructured surfaces. With increase of the cell culture time to 12 hours and 24 hours, the number of attached (and/or spread) stem cells gradually increased. 


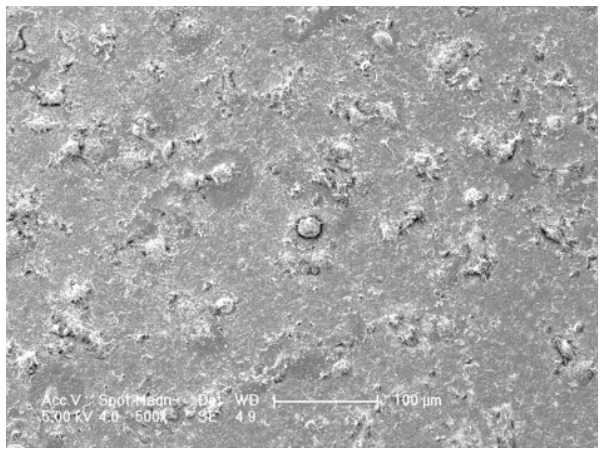

(a)

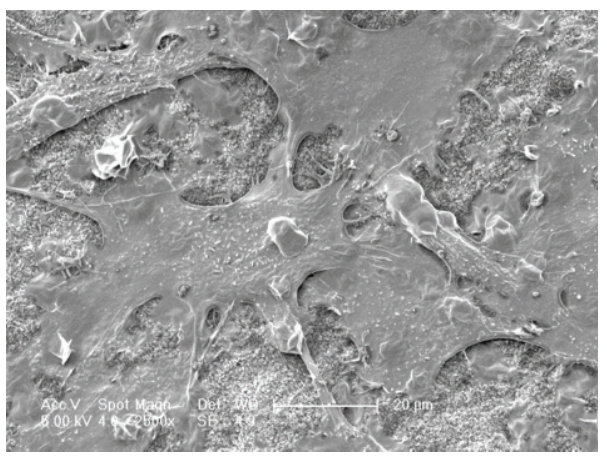

(c)

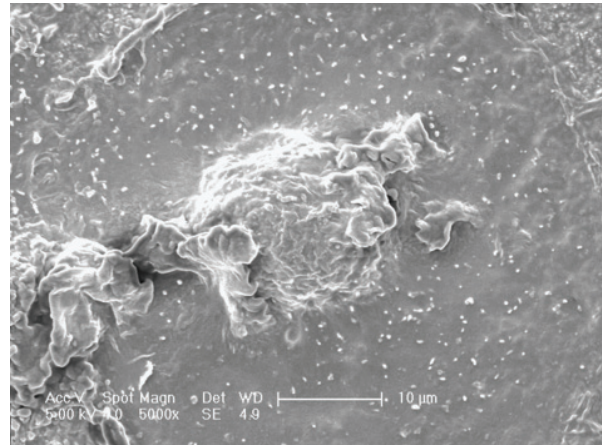

(b)

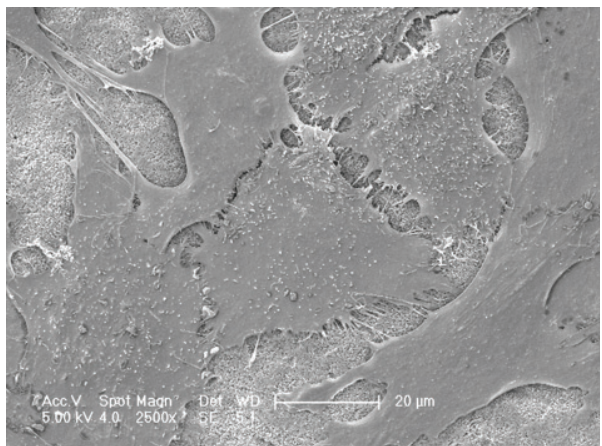

(d)

Figure 6: Attachment of stem cells on the Ti-Nb-Zr-O nanotubes after different culture time (a)-(b) 2 hours, (c) 12 hours, and (d) 24 hours.

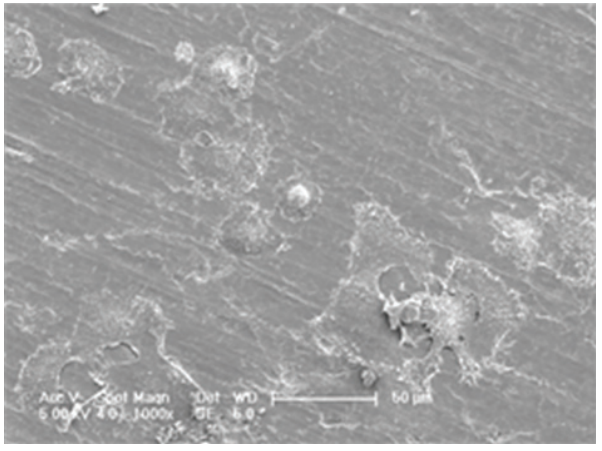

(a)

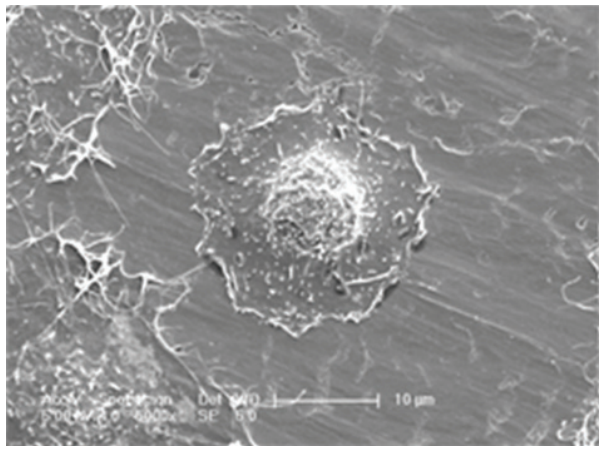

(b)

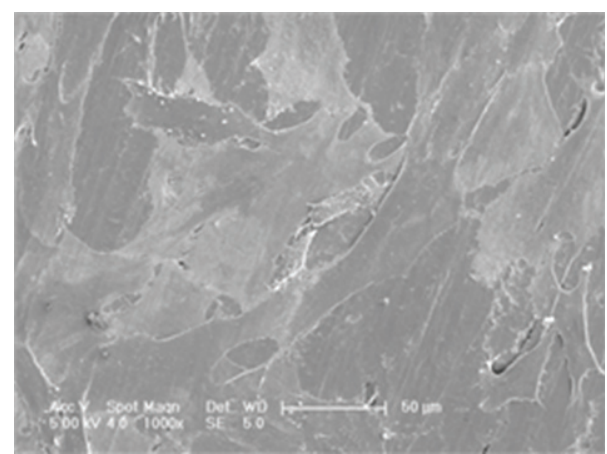

(c)

FIGURE 7: Attachment of stem cells on the pure Ti plate after different culture time (a)-(b) 2 hours and (c) 24 hours. 
The shapes of cell adhesion transformed from process to flat with increase of the stem cell culture time from $2 \mathrm{~h}$ to 12 and $24 \mathrm{~h}$. And the spreading-out tendency of the attached stem cells became more and more obvious with the increase of the cell culture time. As shown in Figure 6(c), after a $12 \mathrm{~h}$ cell culture, the cell adhesion was so evident that most of the stem cells presented a flat shape, which covered much more areas than those for the short-time cultured samples. After $24 \mathrm{~h}$ of cell culture, the spreading out of the attached stem cells was so strong that nearly all of the surfaces of nanostructured surfaces were covered with stem cells, which had flat shapes and numerous filopodia (Figure 6(d)). Obviously, the Ti$\mathrm{Nb}-\mathrm{Zr}-\mathrm{O}$ nanostructures could remarkably facilitate quick cell adhesion and spreading on the oxide surfaces.

Compared to the MSC/Ti-Nb-O nanotube system [14], there was no much difference in all above-mentioned aspects including the cell adhesion rate, the spreading out of attached stem cells, the flat shapes and numerous filopodia after longtime culture. The formation speed of extracellular matrix (ECM) in the present MSC/Ti-Nb-Zr-O system is so quick that all of the cell-free $\mathrm{Ti}-\mathrm{Nb}-\mathrm{Zr}-\mathrm{O}$ nanostructure areas were completely covered with ECM type materials after a shorttime culture of only $2 \mathrm{~h}$. Such a rapid ECM formation is expected to be favorable for fast cell signaling and abundant nutrient reserves.

Figure 7 presents SEM images of stem cells attached on the Ti-O nanostructures after different culture times. In comparison with the MSC/Ti-Nb-Zr-O system, slower formation of ECM in the MSC/Ti-O system could be found. The stem cell response to the Ti-Nb-Zr-O nanostructures seems to be better than that for the undoped Ti-O nanostructures.

\section{Conclusion}

In summary, anodic $\mathrm{Ti}-\mathrm{Nb}-\mathrm{Zr}-\mathrm{O}$ nanostructures were grown on the surface of Ti35Nb5Zr alloy through anodization in $1 \mathrm{M}\left(\mathrm{NH}_{4}\right)_{2} \mathrm{SO}_{4}$ solution containing $0.5 \mathrm{wt} \% \mathrm{NH}_{4} \mathrm{~F}$. The $\mathrm{Ti}-\mathrm{Nb}-\mathrm{Zr}-\mathrm{O}$ nanostructures demonstrated a good in vitro bioactivity of inducing apatite formation and facilitating quick cell adhesion and spreading. For the drug-release system based on gentamicin and the Ti-Nb-Zr-O nanostructures, the release process of the system with a high drug loading content was slower than that with lower drug loading content and it could work more lastingly. The Ti35Nb5Zr alloy modified with Ti-Nb-Zr-O nanostructures has great potential for biomedical applications especially local drug release in the future clinical operation of orthopaedics.

\section{Acknowledgments}

This work was supported by Shanghai Pujiang Program (no. 07pj14047) and 863 Plan of China (2006AA02A1). The authors also thank the support from SEM Lab at Instrumental Analysis Center of SJTU.

\section{References}

[1] D. Q. Martins, W. R. Osório, M. E. P. Souza, R. Caram, and A. Garcia, "Effects of $\mathrm{Zr}$ content on microstructure and corrosion resistance of Ti-30Nb-Zr casting alloys for biomedical applications," Electrochimica Acta, vol. 53, no. 6, pp. 2809-2817, 2008.

[2] X. J. Feng, J. M. Macak, S. P. Albu, and P. Schmuki, "Electrochemical formation of self-organized anodic nanotube coating on Ti-28Zr-8Nb biomedical alloy surface," Acta Biomaterialia, vol. 4, no. 2, pp. 318-323, 2008.

[3] V. S. Saji, H. C. Choe, and W. A. Brantley, "An electrochemical study on self-ordered nanoporous and nanotubular oxide on Ti-35Nb-5Ta-7Zr alloy for biomedical applications," Acta Biomaterialia, vol. 5, no. 6, pp. 2303-2310, 2009.

[4] M. Niinomi, "Mechanical properties of biomedical titanium alloys," Materials Science and Engineering A, vol. 243, no. 1-2, pp. 231-236, 1998.

[5] M. Long and H. J. Rack, "Titanium alloys in total joint replacement-a materials science perspective," Biomaterials, vol. 19 , no. 18 , pp. 1621-1639, 1998.

[6] M. Niinomi, "Fatigue performance and cyto-toxicity of low rigidity titanium alloy, Ti-29Nb-13Ta-4.6Zr," Biomaterials, vol. 24, no. 16, pp. 2673-2683, 2003.

[7] Q. Liu, J. Ding, F. K. Mante, S. L. Wunder, and G. R. Baran, "The role of surface functional groups in calcium phosphate nucleation on titanium foil: a self-assembled monolayer technique," Biomaterials, vol. 23, no. 15, pp. 3103-3111, 2002.

[8] S. H. Oh, R. R. Finõnes, C. Daraio, L. H. Chen, and S. Jin, "Growth of nano-scale hydroxyapatite using chemically treated titanium oxide nanotubes," Biomaterials, vol. 26, no. 24, pp. 4938-4943, 2005.

[9] E. P. Briggs, A. R. Walpole, P. R. Wilshaw, M. Karlsson, and E. Pålsgård, "Formation of highly adherent nano-porous alumina on Ti-based substrates: a novel bone implant coating," Journal of Materials Science: Materials in Medicine, vol. 15, no. 9, pp. 1021-1029, 2004.

[10] J. Park, S. Bauer, K. Von Der Mark, and P. Schmuki, "Nanosize and vitality: $\mathrm{TiO}_{2}$ nanotube diameter directs cell fate," Nano Letters, vol. 7, no. 6, pp. 1686-1691, 2007.

[11] S. Bauer, J. Park, K. V. D. Mark, and P. Schmuki, "Improved attachment of mesenchymal stem cells on super-hydrophobic $\mathrm{TiO}_{2}$ nanotubes," Acta Biomaterialia, vol. 4, no. 5, pp. 15761582, 2008.

[12] K. S. Brammer, S. Oh, J. O. Gallagher, and S. Jin, "Enhanced cellular mobility guided by $\mathrm{TiO}_{2}$ nanotube surfaces," Nano Letters, vol. 8, no. 3, pp. 786-793, 2008.

[13] K. C. Popat, M. Eltgroth, T. J. LaTempa, C. A. Grimes, and T. A. Desai, "Decreased Staphylococcus epidermis adhesion and increased osteoblast functionality on antibiotic-loaded titania nanotubes," Biomaterials, vol. 28, no. 32, pp. 4880-4888, 2007.

[14] D. Ding, C. Ning, L. Huang et al., "Anodic fabrication and bioactivity of $\mathrm{Nb}$-doped $\mathrm{TiO}_{2}$ nanotubes," Nanotechnology, vol. 20, no. 30, Article ID 305103, 2009.

[15] L. Huang, C. Q. Ning, D. Y. Ding et al., "Wettability and in vitro bioactivity of doped $\mathrm{TiO}_{2}$ nanotubes," Journal of Inorganic Materials, vol. 25, no. 7, pp. 775-779, 2010.

[16] K. C. Popat, L. Leoni, C. A. Grimes, and T. A. Desai, "Influence of engineered titania nanotubular surfaces on bone cells," Biomaterials, vol. 28, no. 21, pp. 3188-3197, 2007.

[17] A. Ghicov, S. Aldabergenova, H. Tsuchyia, and P. Schmuki, " $\mathrm{TiO}_{2}-\mathrm{Nb}_{2} \mathrm{O}_{5}$ nanotubes with electrochemically tunable morphologies," Angewandte Chemie, vol. 45, no. 42, pp. 69936996, 2006.

[18] R. Qin, D. Ding, C. Ning, H. Liu, M. Li, and D. Mao, "Fabrication and hydrogen sensing properties of doped titania nanotubes," in Proceedinjgs of the 11th International Conference on Electronic Packaging Technology and High Density Packaging (ICEPT-HDP '10), pp. 1327-1330, August 2010. 
[19] S. Bai, D. Ding, C. Ning et al., "Anodic growth of uniform nanotube arrays on biphase Ti35Nb5Zr alloy," Electrochemistry Communications, vol. 12, no. 1, pp. 152-155, 2010.

[20] L. V. Taveira, J. M. Macák, H. Tsuchiya, L. F. P. Dick, and P. Schmuki, "Initiation and growth of self-organized $\mathrm{TiO}_{2}$ nanotubes anodically formed in $\mathrm{NH}_{4} \mathrm{~F} /(\mathrm{NH} 4)_{2} \mathrm{SO}_{4}$ electrolytes," Journal of the Electrochemical Society, vol. 152, no. 10, pp. B405-B410, 2005.

[21] Y. Li, D. Ding, C. Ning et al., "Thermal stability and in vitro bioactivity of Ti-Al-V-O nanostructures fabricated on Ti6Al4V alloy," Nanotechnology, vol. 20, no. 6, Article ID 065708, 2009.

[22] A. L. Doadrio, E. M. B. Sousa, J. C. Doadrio, J. Pérez Pariente, I. Izquierdo-Barba, and M. Vallet-Regí, "Mesoporous SBA15 HPLC evaluation for controlled gentamicin drug delivery," Journal of Controlled Release, vol. 97, no. 1, pp. 125-132, 2004. 

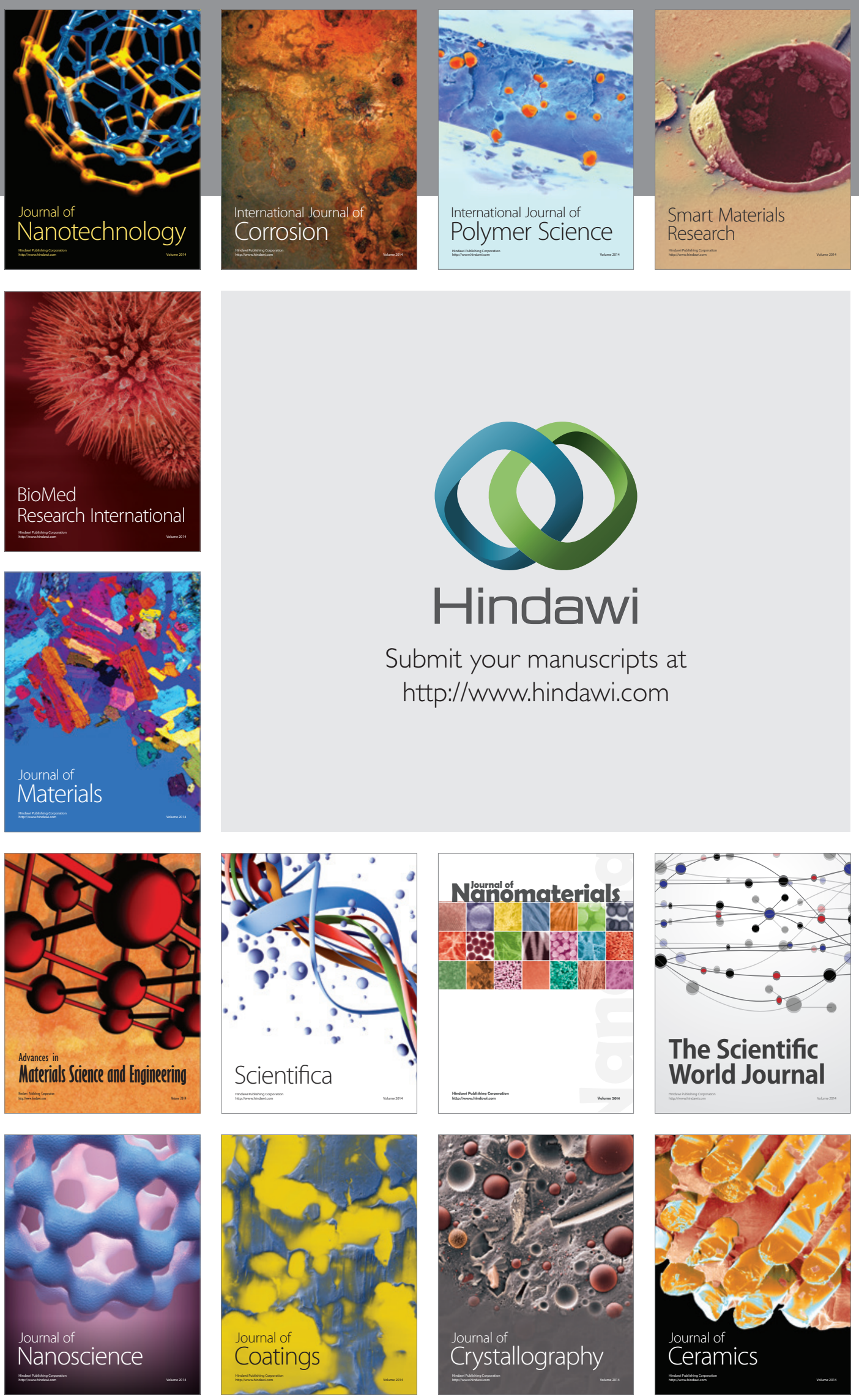

The Scientific World Journal

Submit your manuscripts at

http://www.hindawi.com

\section{World Journal}

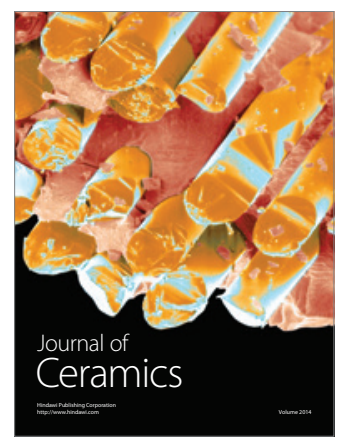

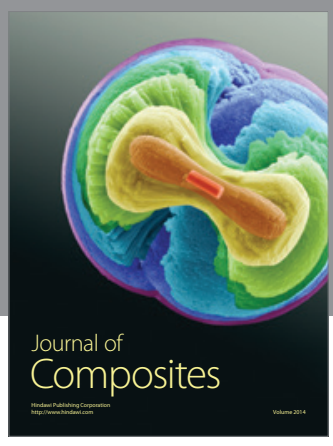
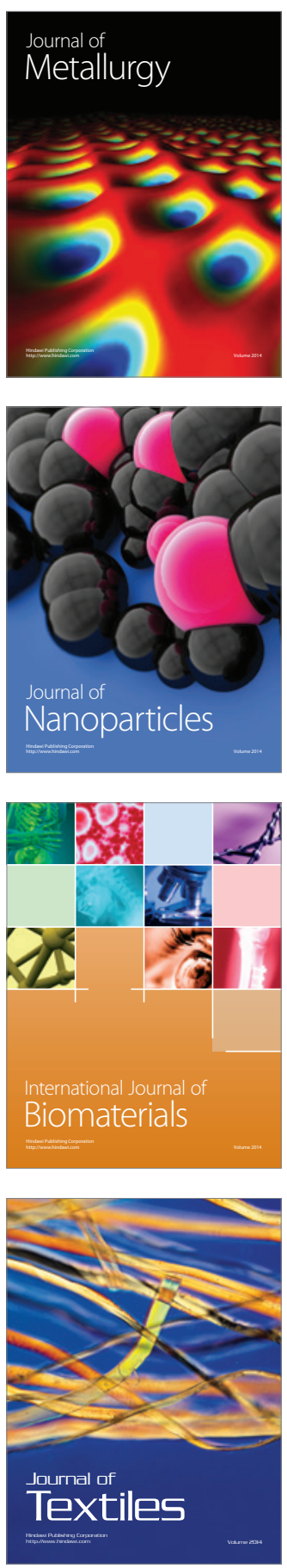